(73\%) with 10 or fewer seizures. When patients discontinued AED treatment, 232 of 331 patients overall $(70 \%)$ remained seizure free. For each pretreatment seizure number greater than one, the number of patients successfully discontinuing medication was the same. Of those treated after a single seizure, $57 \%$ were seizure free after AED discontinuation, compared to $72 \%$ with more than one pretreatment seizure. Patients with more than 10 pretreatment seizures were more likely to have complex partial seizures $(59 \%)$ than those with 10 or fewer seizures (16\%). (Camfield C, Camfield P et al. Does the number of seizures before treatment influence ease of control or remission of childhood epilepsy? Not if the number is 10 or less. Neurology January 1996;46:41-44). (Reprints: Drs Camfield, IWK Children's Hospital, Box 3070, Halifax, Nova Scotia, B3J 3G9, Canada).

COMMENT. These findings tend to disprove the theory that seizures beget seizures, at least in children permitted to have 10 or fewer seizures before treatment with AEDs is begun. The ease of seizure control and frequency of remission are unaltered if medication is delayed for up to 9 recurrences. Children excluded from this study were those with myoclonus, absence, akinetic, and infantile myoclonic seizures, which are too numerous to count. The introduction of antiepileptic treatment after a first or second seizure in children with generalized tonic-clonic or partial seizures requires further evaluation. Each patient must be considered as an individual, and these findings should be weighed in conjunction with past practices when considering advisability of antiepileptic treatment. Some previous studies have found that risk factors for seizure relapse after withdrawal of antiepileptic treatment have included delay in initiation of therapy. See Progress in Pediatric Neurology I, PNB Publishers, 1991, pp100-104.

\title{
AED WITHDRAWAL IN CHILDREN WITH CEREBRAL PALSY
}

The safety of antiepileptic drug (AED) withdrawal in 65 children with cerebral palsy (CP) who had been seizure-free for at least 2 years was investigated in the Neurology Department, Texas Scottish Rite Hospital for Children, Dallas. Seizure relapses occurred in 27 patients $(41 \%)$. Those with spastic hemiparesis had the highest relapse rate $(61 \%)$ and spastic diplegia was associated with the lowest rate (14\%). Mental subnormality, epileptiform EEG abnormality, type of CT abnormality, family history of epilepsy, mono or polytherapy, and gender were not correlated with risk of seizure relapse. (Delgado MR et al. Discontinuation of antiepileptic drug treatment after two seizure-free years in children with cerebral palsy. Pediatrics February 1996;97:192-197). (Reprints: Dr MR Delgado, Texas Scottish Rite Hospital for Children, 2222 Welborn St, Dallas, TX 75219).

COMMENT. Despite abnormal neurologic examinations, almost two thirds of these CP patients remained free from seizures for periods of at least 2 years after AED withdrawal. Patients with spastic hemiparesis have the highest relapse rate, and drug withdrawal should be discouraged or attempted only with great caution.

\section{SPECT AND EEG IN LANDAU-KLEFFNER SYNDROME}

Five right-handed children with Landau-Kleffner syndrome (LKS) were studied with EEG and single-photon emission computed tomography (SPECT) before and after 6 months of corticosteroid therapy at the Universities of Estadual de Campinas and Sao Paulo, Brasil. EEGs showed both focal and 
generalized spikes, and spike-wave bitemporal discharges. MRI was normal. Brain SPECT showed abnormal perfusion in the left temporal lobe. Steroids and AEDs had no significant beneficial effects on either aphasia or behavior. (Guerreiro MM et al. Brain single photon emission computed tomography imaging in Landau-Kleffner syndrome. Epilepsia Feb 1996;37:60-67). (Reprints: Dr MM Guerreiro, Rua Camargo Paes 637, 13073-350 Campinas, Sao Paulo, Brazil).

COMMENT. Landau-Kleffner syndrome is an acquired epileptic aphasia or verbal auditory agnosia affecting children between 2 and 5 years of age and characterized by profound language dysfunction, seizures, and/or a paroxysmal EEG abnormality, and associated with a generally poor prognosis. The EEG abnormality is frequently bilateral and localization of a focal temporal lesion is often difficult. Brain SPECT in the above study, and previously reported by Morrell et al, may show hypoperfusion of the left temporal cortex.

Subpial intracortical transection in L.KS. Morrell and colleagues at Rush-Presbyterian-St Luke's Medical Center and Epilepsy Center, Chicago, describe a methohexital suppression test which permits epileptiform potentials to stand out in an otherwise flat EEG. Using this technique to define a unilateral origin for the bilateral epileptiform discharge, 14 children with LKS were treated surgically by subpial intracortical transection. Eleven (79\%) are now speaking, and 7 of these no longer require speech therapy. This method of treatment in selected cases appears superior to corticosteroids which have at best a temporary beneficial effect. (Morrell F et al. LandauKleffner syndrome. Treatment with subpial intracortical transection. Brain 1995;118:1529-1546).

\section{VACCINE-RELATED NEUROLOGIC DISORDERS}

\section{PERTUSSIS VACCINE CNS SEQUELAE REASSESSMENT}

The role of whole-cell pertussis vaccine as a cause of permanent neurologic damage has been reassessed by the American Academy of Pediatrics, Committee on Infectious Diseases, in light of new 10-year follow-up findings of the National Childhood Encephalopathy Study (NCES) in Great Britain. Although causal relationship is not definitely acknowledged, the committee now concludes that DTP vaccination can be associated with chronic neurologic dysfunction in children who had severe acute neurologic illnesses after DTP vaccination. The immunization guidelines in the 1994 Red Book continue to be recommended, but specific guidelines for acellular pertussis vaccines will be revised after FDA approval of new products. (AAP Committee on Infectious Diseases Report. The relationship between pertussis vaccine and central nervous system sequelae: continuing assessment. Pediatrics February $1996 ; 97: 279-281)$.

COMMENT. Pediatric neurologists caring for patients with increased susceptibility to precipitation or exacerbation of neurologic disorders such as seizures, behavior disorders, or language and developmental delays should use caution in advising parents concerning risks of immunizations. The AAP committee is careful to qualify their recommendations by a disclaimer rider.

Varicella vaccine-induced acute cerebellar ataxia is reported for the first time, affecting a 2-year-old boy who developed vomiting and ataxia 10 days after vaccination. MRI showed multiple demyelinating lesions. Recovery followed in 3 weeks. (Sunaga Y et al. Pediatr Neurol 1995;13:340-342). 